
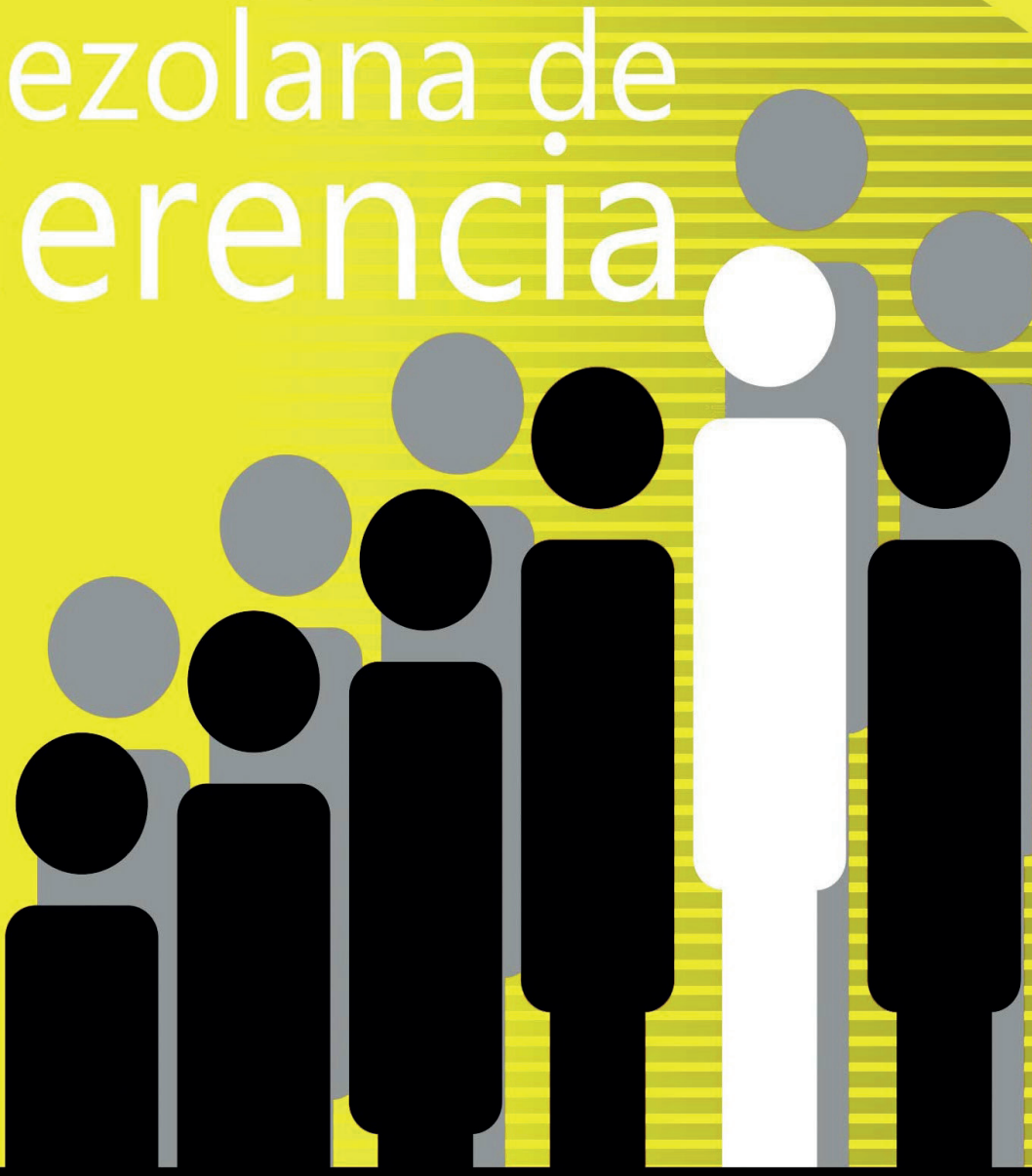


\title{
Diferencias en los determinantes del éxito en el emprendimiento en México, una perspectiva de género
}

\author{
Sánchez Tovar, Yesenia* \\ Macías García, Miguel Ángel $\dagger^{* *}$ \\ Mendoza Flores, José Esteban ${ }^{* \star *}$
}

\section{Resumen}

En las últimas décadas las mujeres han logrado posicionarse en los mercados laborales y además participar de manera activa en la creación de nuevas empresas (Langowitz y Minniti, 2007). En el caso de México, de acuerdo con datos de la Secretaría de Hacienda, 3 de cada 5 negocios que se abren son dirigidos por mujeres, del mismo modo se ha observado que en este país, el gobierno ha puesto énfasis en generar programas de apoyo para las mujeres emprendedoras. No obstante, el índice de fracaso de los emprendimientos es del $75 \%$. Lo cual obliga a investigar los factores que determinan el éxito de los emprendimientos entre hombres y mujeres. Para ello, se analizan los factores conductuales y gerenciales que determinan el impacto en el éxito emprendedor, realizando un estudio empírico a 255 directivos de empresas de reciente creación (de 0 a 3 años de apertura) en cuatro ciudades del norte de México. A través del uso de ecuaciones estructurales se pudieron observar las diferencias en el éxito en los negocios entre hombres y mujeres. En primer lugar, se observó que lo que determina el éxito de las mujeres en los negocios radica en los elementos conductuales, es decir, son los rasgos psicológicos (extraversión, apertura y conciencia). Mientras que para los hombres las capacidades gerenciales son las que determinan su éxito. Los resultados anteriores, dejan ver la necesidad de fortalecer la capacitación gerencial en las mujeres para alcanzar el éxito empresarial.

Palabras clave: Emprendimiento exitoso; género; rasgos de la personalidad.

\section{Recibido: 15.11.20 Aceptado: 10.02.21}

Doctora en Economía y Gestión de las Organizaciones. Profesora Investigadora en la Facultad de Comercio y Administración Victoria de la Universidad Autónoma de Tamaulipas E-mail:yesanchez@docentes.uat.edu. mx. (Autor para la correspondencia). ORCID: https://orcid.org/0000-0002-4299-191X

** Doctor en Ciencias Administrativas. Profesor Investigador de la Universidad Autónoma de Tamaulipas. E-mail:amacias@docentes.uat.edu.mx ORCID: https://orcid.org/0000-0003-1151-8131

*** Maestro en Administración. Profesor Investigador de la Universidad Autónoma de Tamaulipas. E-mail:emflores@docentes.uat.edu.mx ORCID: https://orcid.org/0000-0002-1255-3214 


\title{
Differences in the Determinants of Entrepreneurship Success In Mexico, A Gender Perspective
}

\begin{abstract}
In Mexico, according to data from the Ministry of Finance, 3 out of 5 businesses that are opened are run by women, in the same way it has been observed that in this country, the government has placed an emphasis on generating support programs for enterprising women. However, the failure rate of the enterprises is $75 \%$. This forces to investigate the factors that determine the differences in the success of the enterprises between men and women. To do this, the behavioral and managerial factors that determine the impact on entrepreneurial success are analyzed, conducting an empirical study of 255 managers of recently created companies (from 0 to 3 years of opening) in four cities in northern Mexico. Using structural equations model, it was obtained that behavioral elements are entrepreneurship success determinants of women, that is, extraversion, openness and awareness. While for men, managerial skills are what determine their success. The previous results show the need to strengthen managerial training in women to achieve business success.
\end{abstract}

Keyword: Successful entrepreneurship; gender; personality traits.

\section{Introducción}

En las últimas décadas el emprendimiento femenino ha tenido un crecimiento importante en todo el mundo (Runyan, Huddleston y Swinney, 2006), pero no es sólo el crecimiento en términos cuantitativos, sino también de manera cualitativa se ha constatado la progresiva presencia de las mujeres a nivel mundial (Minitti, 2009).

La reciente incursión intensiva de la mujer en el emprendimiento pone a este tema en el foco de las recientes investigaciones en el ámbito de la administración, debido a ello, es de suma importancia conocer los factores que determinan no sólo el emprendimiento sino el éxito de las nuevas empresas, que algunos autores definen de manera cuantitativa por medio de indicadores económicos / financieros, incluyendo rentabilidad, productividad o tasa de crecimiento, una posición competitiva favorable que conduce a un rendimiento económico superior y sostenible (aumento o mantenimiento de la cuota de mercado de la empresa) (Wiklund y Shepherd, 2005), los ingresos, la riqueza personal y el volumen de negocios (Amit, et al, 2000; Perren, 1999).

Mientras que los factores cualitativos citados con frecuencia incluyen la capacidad de innovación, la satisfacción del empleado / cliente o del emprendedor, y el crecimiento de la empresa (Covin, Green y Slevin, 2006; Hill y Jones, 2011).

Es así que toma importancia investigar los factores individuales 
que permean en los emprendedores y analizar cuáles de estas características los hacen ser más exitosos (Naktiyok, Karabey y Gulluce, 2010). En este sentido es importante analizar las características individuales del emprendedor, debido a que es él quien con sus rasgos y habilidades pueden llevar al éxito su nuevo negocio (Brandstätter, 2011; Leutner, et al, 2014). En los últimos años la definición de emprendimiento no se queda sólo con el nacimiento de nuevas empresas, si no que incluye el enfoque individual sumándose cuestiones de actitud, como una manera de razonar, analizar y actuar en búsqueda de oportunidades, haciendo uso de características personales como el liderazgo y la capacidad de catalizar los cambios para la creación de empresas (Abu-Saifan, 2012).

La literatura en esta línea ha mostrado dos enfoques para abordar la visión individual del emprendimiento, el primer enfoque es el conductual que estudia los rasgos psicológicos que debe poseer el emprendedor los cuales determinan el nivel de compromiso con la iniciativa empresarial (McClelland, 1961) y el enfoque gerencial que evalúa las competencias que se deben tener para convertirse en un emprendedor, dichas habilidades afectan en el resultado de la empresa (Veciana, 1999).

Sobre el enfoque conductual se admite que los directivos tienen características psicológicas que definen su identidad con base a sus actitudes, valores y motivaciones que se definen a través de sus experiencias $y$ que determinan su nivel de emprendedurismo, personas con rasgos como estabilidad emocional y necesidad de logro las vuelve más propensas a emprender (Bulut, et al, 2010).

Autores como D’Enbeau, Villamil, y Helens-Hart (2015) y Munkejord, (2017), definen que existen estereotipos basados en el género en relación a los determinantes del emprendimiento, debido a que se espera que las mujeres cumplan con roles familiares lo que vuelve difícil su inclusión en el mundo empresarial, además que el modelo emprendedor tiene rasgos de masculinidad. En esta investigación se estudia si los factores conductuales inciden en el éxito del emprendimiento diferenciando por género su efecto.

Por otro lado, desde un enfoque gerencial se establece que las personas que emplean habilidades gerenciales como comunicación abierta y liderazgo dinámico pueden tener resultados favorables en el emprendimiento de sus empresas (Sunindijo et al, 2007), este tipo de habilidades le permite al directivo sobrellevar la incertidumbre de un entorno empresarial dinámico (Ensley, Pearce y Hmieleski, 2006).

Aunado a lo anterior, existen investigaciones que determinan que un emprendedor cualificado tiene mayores posibilidades de éxito, además dichas habilidades y conocimientos son adquiridos a través de la experiencia empresarial la cual favorece el potencial emprendedor (Schefczyk, 2001; Minniti y Bygrave, 2001, Aubert et al, 2006).

En cuanto a las diferencias por género, existen diferentes estudios que indican que las mujeres encuentran más barreras que los hombres para acceder a apoyos financieros que les permitan tener crecimiento en sus empresas nacientes hombres (Langowitz y Minniti 2007; Minniti y Nardone 2007). Destacando a su vez que la personalidad y las habilidades de hombres y mujeres emprendedoras no son homogéneas. Debido a lo anterior, la presente investigación tiene por 
objetivo analizar los determinantes del emprendimiento exitoso a través de los enfoques gerencial y conductual, diferenciando estos factores por género en el norte de México, con la finalidad de apoyar a la identificación de los elementos que deben estimularse para que los individuos a partir de su género logren el emprendimiento exitoso. Para la comprobación de este objetivo, se desarrolló una investigación cuantitativa de alcance causal a través del uso de ecuaciones estructurales por el método de covarianzas.

La población objeto de estudio estuvo conformada por los empresarios de negocios de reciente creación de la región de Tamaulipas en el norte de México integrada por 24,460 negocios con una antigüedad menor a 3 años, logrando a partir de dicha población una muestra de 255 emprendedores.

Para medir los rasgos de los factores conductuales del emprendedor, se utilizó la escala de European Certificate in Entrepreneurship ("ECENT", 2006), en tanto que las habilidades gerenciales $y$ de conocimiento fueron medidas a través de la escala presentada por Formichella (2004) y Acs (2008). El éxito emprendedor fue medido por la escala desarrollada por Wang (2008) y Shepherd, et al, (2010).

\section{Determinantes personales del emprendimiento exitoso}

Según plantea Montes de Oca (2020), el emprendimiento deriva de la convergencia de varios factores entre los que destacan el capital en todos sus tipos, su devenir histórico, los instrumentos de reproducción y sus relaciones, las diferentes fuerzas que subyacen en las diferentes situaciones de emprendimiento y sus influjos, así como el factor humano- social que es preponderante.

En esta investigación, el estudio del emprendimiento puede ser abordado desde dos perspectivas, la de los determinantes asociados con el individuo y los relacionados con el entorno. En esta investigación se centra la atención en conocer los determinantes asociados con el individuo. Como se mencionó en la introducción el estudio de los determinantes personales puede abordarse desde dos perspectivas teóricas, la del enfoque conductual y del enfoque gerencial. En este apartado se desarrollan los preceptos teóricos anteriores.

\subsection{Enfoque psicológico conductual del emprendedor}

El enfoque conductual se ha centrado en el estudio de los rasgos psicológicos y características personales que permiten al individuo convertirse en emprendedor (Bygrave y Hofer, 1992, Bulut, 2010), según este enfoque son las capacidades y la personalidad del individuo determinantes de la nueva empresa (Herron y Robinson, 1993; Fayolle, Linán y Moriano, 2014).

Dentro de las teorías de psicológicas, una de las más utilizadas en diferentes investigaciones para explicar la personalidad del emprendedor es la teoría de los cinco grandes rasgos, desarrollada por Goldberg en 1992 (Leutner, et al, 2014; Şahin, Karadağ, y Tuncer, 2019; Murugesan y Jayavelu, 2017). Así mismo, la motivación actúa como un agente psico-conductual que impulsan a una persona a emprender. A continuación, se profundiza en los rasgos de la personalidad y los motivantes personales que influyen en el emprendimiento exitoso. 


\subsubsection{Modelo de los cinco grandes rasgos de la personalidad}

Las predisposiciones conductuales que el individuo sigue ante determinadas situaciones fueron denominadas rasgos de la personalidad y después de décadas de estudio, en los 90's se definieron 5 grupos de rasgos que definen la personalidad de cualquier sujeto (Romero, 2005). Dichos rasgos identificados fueron la extroversión, apertura, estabilidad emocional, agradabilidad y conciencia (Goldberg, 1992). Los rasgos de la personalidad son asociados con diferentes acciones - conductas del individuo como el emprendimiento (Leutner, et al, 2014; Şahin, Karadağ, y Tuncer, 2019)

Siguiendo con el modelo de los cinco grandes se presenta la extroversión, dicho rasgo implica a las personas sociables, asertivas y positivas emocionalmente, generalmente son confiados, comunicativos y enérgicos (Watson y Clark, 1997; John et al, 2008), estas características son necesarias para desenvolverse en el entorno empresarial.

Otro de los rasgos del modelo en cuestión, es la apertura cuya definición propuesta por Carlisle y Flynn (2005) quienes explican que la apertura es la disposición de las personas para adecuar sus creencias, actitudes y comportamientos cuando se exponen a nuevas ideas, situaciones y experiencias (Carlisle y Flynn, 2005). Desde una perspectiva del entorno, las personas con altos niveles de apertura a la experiencia han sido consideradas cultas, curiosas, imaginativa y de mente abierta (Barrick, Mount y Judge, 2001)
Una tercera dimensión de este modelo es la conciencia como un amplio dominio de rasgos que incluye múltiples facetas: trabajo duro, orden, control de impulsos, confiabilidad y convencionalidad. La conciencia también se asocia con numerosos comportamientos, como terminar una tarea a tiempo, llegar a una reunión a tiempo, etc. En la escala de este autor, la conciencia se entiende como el rasgo de responsabilidad de perseverancia en la consecución de los objetivos propios, la concentración en los logros y la preservación de sus propias reglas y estándares (John et al, 2008; Jackson, et al, 2010).

Otro factor del modelo de los cinco grandes es la estabilidad emocional, las personas emocionalmente estables tienen puntos de vista positivos sobre sus actividades y sobre las demás personas, y son relajados (Sung y Choi, 2009). Por otro lado, el puntaje alto en neuroticismo explica las reacciones emocionales a los sentimientos negativos, como la ira y la ansiedad, por lo que la puntuación baja explica la estabilidad emocional (Migliore, 2011).

Por último, la agradabilidad como parte del modelo de los cinco grandes se representa con emociones como la bondad, el afecto y la humildad. Aquellos que puntúan alto en la amabilidad generalmente son personas muy morales y sinceras. Tienden a ser más cooperativos que combativos $y$ suavizarán las cosas por el bien de la paz. Aquellos que obtienen un puntaje bajo en amabilidad pueden ser muy competitivos, a veces manipuladores, y colocar su propio interés antes de llevarse bien con los demás (Anderson et al, 2018). 


\subsubsection{Motivación de logro}

La motivación de logro es una de las características conductuales presentes en la personalidad del emprendedor (Veciana, 2005). De acuerdo con McClelland (1965), esta motivación se traduce en la necesidad de una persona para desarrollar actividades desafiantes y que traen consigo una sensación de éxito (Dinur, 2011). La motivación del fundador de la empresa representa el compromiso de éste con su proyecto o idea de empresa, por lo que en función de su naturaleza e intensidad estaremos ante una empresa con futuro o sin él (Barba, 2000).

Por otro lado, la motivación dentro del enfoque conductual se presenta la relación de la motivación de logro como favorecedor de la actividad emprendedora (Van Riel et al, 2004; Veciana, 2005; Pires et al, 2008; Chell, 2008; Shepherd, 2010; Dinur, 2011), esta característica permite un mayor apego del individuo con el emprendimiento. La motivación de logro no debe confundirse con los rasgos de la personalidad, debido a que esta es una característica conductual que debe estar presente para que el emprendedor alcance su cometido.

\subsubsection{El enfoque conductual y la diferencia por género}

El emprendimiento y la conducta psicológica del emprendedor pueden presentar diferencias cuando se trata del género, en otras palabras, la personalidad de las mujeres emprendedoras es distinta de los hombres emprendedores (Lucas y Donnellan, 2011; Roberts y DelVecchio, 2000; Blonigen et al, 2008) y a pesar de que la legislación y las políticas de fomento de la igualdad de género, impulsan avances hacia una mayor igualdad de las mujeres, estos continúan siendo lentos en algunos sectores en particular (De Miguel Pascual; ParrattFernández, y Berganza, 2019).

El conocer si el género condiciona una conducta psicológica que lleve al emprendimiento resulta importante para definir qué tipo de actividades, talleres o programas de formación deben desarrollarse para que el hombre o la mujer logren una personalidad que los acerque a un emprendimiento exitoso (Murugesan y Jayavelu, 2017).

Analizando los rasgos de la personalidad, encontramos trabajos como los de Lucas y Donnellan (2011) y Roberts y DelVecchio (2000), quienes implicaron que el género no determina la personalidad del emprendedor; sin embargo, Blonigen et al, (2008) encontró que el rasgo de agradabilidad es más fuerte en las mujeres emprendedoras que en los hombres.

Otros estudios empíricos como los de Roberts et al, (2006) y Robins, Fraley, Roberts y Trzesniewski (2001) muestran que las mujeres obtienen puntuaciones altas de neuroticismo y extroversión y bajos niveles de apertura, conciencia y agradabilidad.

En cuanto a la motivación al logro, algunos estudios han destacado que la motivación afecta de forma distinta a hombres y mujeres, lo que conlleva a desigualdad en los niveles de emprendimiento (Sexton y BowmanUpton, 1990). En España, se ha analizado que las inversiones efectuadas por mujeres son más prudentes, que las que han realizado de los hombres (GonzálezMorales, 2001). Existe alguna prueba de que una de las principales razones para crear empresas por parte de las mujeres emprendedoras es la búsqueda de logro (Marlow, 1997). 
Las diferencias entre hombres y mujeres se suelen atribuir a causas como la estructura biológica de cada género, así como el rol que juegan culturalmente tanto hombres como mujeres (Busato, et al, 2000) por lo que se requiere un estudio riguroso que identifique si los determinantes del emprendimiento son distintos entre hombres y mujeres (Murugesan y Jayavelu, 2017).

A partir del enfoque conductual se plantean las siguientes hipótesis:

H1: Los rasgos personales favorece el éxito emprendedor de hombres y mujeres

H2: La motivación de logro favorece el éxito emprendedor de hombres y mujeres

\subsection{Enfoque gerencial}

Para lograr el emprendimiento exitoso, el individuo tiene que conseguir una combinación entre sus características psico-conductuales asociadas a su personalidad y las características adquiridas relacionadas con sus habilidades o capacidades empresariales (Veciana, 2005). El enfoque gerencial es la base para la creación de empresas (Ihrig et al, 2006), y en él se plantea que el emprendedor debe sentir que posee los conocimientos y las habilidades necesarias para lograr la creación de una empresa.

Siguiendo esta idea, Minniti y Bygrave, (2001) y Cuervo, Ribeiro y Roig (2007) argumentan que es necesario que el emprendedor cuente con la educación, experiencia, conocimientos y habilidades necesarios que le permitan aumentar su confianza para aventurarse a crear la nueva empresa, logrando un papel único y crítico en la economía de un territorio. En este apartado se explican la importancia de las habilidades gerenciales y el conocimiento del entorno, como elementos del enfoque gerencial necesarios para el emprendimiento.

\subsubsection{Habilidades gerenciales}

Por otra parte el enfoque gerencial (Veciana, 1999), sugiere la distinción de habilidades y conocimientos empresariales fundamentales que deben poseer los emprendedores (Codina, 2005), diferentes trabajos resaltan que las PYMES son más exitosas donde se les da mayor importancia a las habilidades y conocimientos de gestión (Acs y Audretsch, 1990; Laforet, 2013), esto le permite al empresario obtener un stock de características gerenciales que lo animarán a crear empresas (Minniti y Bygrave, 2001).

Del mismo modo, al hablar del enfoque gerencial como el cúmulo de características empresariales, debe considerarse una dimensión adicional que es la experiencia empresarial, laboral o de gestión, lo que permite una mejor relación con proveedores y clientes (Kolb, Osland, Rubin, 2007), también Cuervo, Ribeiro y Roig (2007) explican el papel único y crítico del empresario en la economía, sus argumentos encajan perfectamente en una teoría gerencial de la formación empresarial.

\subsubsection{Conocimiento del entorno}

Para terminar con la revisión de la literatura se presenta un factor que pertenece al enfoque gerencial, en este sentido existen distintos elementos que moldean el entorno emprendedor, tales como aspectos políticos, legalesregulatorios, sociales, demográficos, económicos, entre otros, que ejercen una influencia en las organizaciones y 
en las personas involucradas en ellas, desde la creación de nuevas empresas hasta su desenvolvimiento, de forma positiva o negativa dependiendo de sus características (Acs y Amoros, 2008; Reisinger y Lehner, 2015; SaavedraGarcía, Tapia-Sánchez, y Aguilar-Anaya, 2015).

Considerando un entorno que es dinámico y volátil, el gobierno establece regulaciones impositivas que dan facilidades para la apertura de empresas y también en la etapa de crecimiento de la misma (Clarke et al, 2016), de esta manera las regiones en un país son generalmente heterogéneas y permiten diferentes condiciones para crear empresas, el conocimiento de estas condiciones favorece la creación, puesta en marcha y crecimiento de la empresa (Hajek, Nekolova y Novosak, 2015), además, comprender los factores que promueven o detienen el nacimiento de nuevas empresas es importante para los esfuerzos para la creación de empresas que varía dependiendo del contexto institucional y del nivel de desarrollo económico (Acs, Desai y Klapper, 2008).

Una vez revisados los factores gerenciales que determinan el éxito del emprendimiento se proponen las siguientes hipótesis.

H3: Las habilidades y la experiencia gerencial favorece el éxito emprendedor de hombres y mujeres.

H4: El conocimiento del entorno empresarial favorece el éxito emprendedor de hombres y mujeres.

\section{Consideraciones metodológicas de la investigación}

Para la contrastación de las hipótesis planteadas se desarrolló una investigación cuantitativa de alcance causal. La población sujeta de estudio estuvo conformada por los empresarios de unidades económicas con una antigüedad menor a 3 años que identifica a un negocio en etapa emprendedora, y que se ubican en la región de Tamaulipas en el norte de México. El total de la población es de 24,460 negocios de acuerdo con el Instituto Nacional de Estadística y Geografía (INEGI, 2016). Se calculó una muestra representativamente estadística con un nivel de confianza del $95 \%$ y un margen de error del $5 \%$, obteniendo una muestra calculada de 379 empresas de reciente creación, logrando una tasa de respuesta del $67.38 \%$ de la muestra, es decir 255 empresas encuestadas.

Para la recolección de datos se diseñó una encuesta que capturaba las variables sujetas de estudio a través de una escala tipo Likert de 5 puntos que va desde un $1=$ totalmente desacuerdo a un $5=$ totalmente de acuerdo. La encuesta estuvo basada en escala para la evaluación del emprendedor (European Certificate in Entrepreneurship "ECENT", 2006), ésta evalúa las características de los factores conductuales que identifican los rasgos de la personalidad y la motivación al logro. En tanto que las habilidades gerenciales y el conocimiento de entorno se midieron a través de 8 ítems basados en Formichella (2004) y Acs (2008). Por otro lado, el éxito del emprendimiento fue medido a través de 8 ítems de tipo financiero y no financiero basado en autores como Shapero (1984), Wang (2008) y Shepherd, et al, (2010).

Para realizar el análisis estadístico se empleó la técnica multivariante ecuaciones estructurales por covarianzas por medio del paquete estadístico AMOS y SPSS versión 21. Los modelos 
por ecuaciones estructurales permiten analizar múltiples relaciones causales (Hair et al, 2010), siendo lo adecuado para el modelo de investigación planteado, asimismo, se utilizó un análisis multigrupo que permite dividir la muestra a partir de una característica cualitativa determinada. En este caso la variable cualitativa utilizada fue el género del emprendedor, masculino y femenino.

La evaluación del modelo de medida permite realizar la validación de la escala, para ello que se llevó a cabo los análisis de validez interna, validez convergente y validez discriminante, enseguida se presentan los resultados que arrojaron dichas pruebas.

La validez interna consiste en evaluar la validez de cada variable inmersa en el trabajo, en este sentido se obtuvieron los pesos de regresión estandarizados por medio del análisis factorial confirmatorio (CFA), en esta etapa se eliminan los ítems con cargas factoriales menores a 0.5 (Hair, et al, 2010), resultando afectadas dos dimensiones de los cinco grandes rasgos de la personalidad que fueron agradabilidad y estabilidad emocional, por lo que fueron eliminadas.

Por otro lado, del factor de motivación se perdieron 2 ítems, 2 de extroversión, 1 de apertura, 2 de éxito del emprendimiento, 1 de conocimiento de entorno y 1 de conocimiento. En la tabla 1 se muestran la fiabilidad interna de las variables de la personalidad, apertura, conciencia y extroversión, de la variable motivación, de las habilidades gerenciales, la experiencia y el conocimiento del entorno, además de la variable de éxito emprendedor cuyos valores son satisfactorios, es decir superiores a 0.5 .

\section{Tabla 1}

Fiabilidad interna de los constructos

\begin{tabular}{llll}
\hline Variable & Indicador & Definición de indicador & $\begin{array}{l}\text { Pesos de regresión } \\
\text { estandarizados }\end{array}$ \\
\hline Motivación de logro & MO1 & Planificación profesional & 0.818 \\
& MO2 & Relaciones profesionales & 0.784 \\
\hline \multirow{2}{*}{ Apertura } & AP1 & Entornos creativos & 0.728 \\
& AP2 & Originalidad & 0.931 \\
\hline Extroversión & AP3 & Creatividad & 0.818 \\
\hline \multirow{2}{*}{ Eonciencia } & EV4 & Diálogo continuo & 0.572 \\
& CO1 & Sociabilidad & 0.831 \\
\hline & CO2 & Compromiso con la tarea & 0.908 \\
& CO3 & Eficiencia en la tarea & 0.879 \\
& & Eficacia en la tarea & 0.716 \\
\hline
\end{tabular}


pp. $880-902$

Revista Venezolana de Gerencia, Año 26 No. 94, 2021

\section{Cont... Tabla 1}

\begin{tabular}{llll}
\hline $\begin{array}{lll}\text { Habilidades Geren- } \\
\text { ciales }\end{array}$ & II1 & Interés en novedades & 0.633 \\
& II2 & Aporte de conocimientos & 0.756 \\
& II3 & Entusiasmo & 0.785 \\
& EL2 & Experiencia en negocios & 0.580 \\
& CA1 & Ideas convincentes & 0.803 \\
\hline $\begin{array}{l}\text { Conocimiento del en- } \\
\text { torno }\end{array}$ & CE4 & Nivel de desarrollo & 0.842 \\
& CE3 & Oportunidad creación emp & 0.726 \\
\hline \multirow{3}{*}{ Éxito Emprendedor } & EX5 & Margen de utilidad & 0.872 \\
& EX4 & Rentabilidad financiera & 0.809 \\
& EX3 & Flujo de caja & 0.761 \\
& EX6 & Utilidad operacional & 0.864 \\
& EX7 & Ratio de ventas & 0.871 \\
& EX8 & Retorno sobre inversión & 0.772 \\
\cline { 2 - 3 } & & &
\end{tabular}

Fuente: Elaboración propia mediante Amos 21.0

Una vez analizada la fiabilidad de la escala se procede a evaluar la validez convergente y discriminante que componen la fiabilidad compuesta.

Para medir la homogeneidad de las puntuaciones de los ítems que pertenecen a cada constructo, se realiza el análisis de confiabilidad convergente o consistencia interna de la escala. Además, se mide la rigurosidad con la que se están las variables manifiestas a la misma variable latente. Para llevar a cabo dicho análisis se emplean los índices del coeficiente del alfa de Cronbach $(\alpha)$ y el coeficiente omega $(\omega)$ (Heise y Bohrnstedt 1970), además de la varianza media extraída (AVE).
Así los valores aceptables para el coeficiente del alfa de Cronbach deben superar el umbral de 0.7 , el coeficiente omega debe estar dentro del rango de 0.7 y 0.9 , por último, la varianza media extraída debe superar el valor de 0.5 como indican Fornell y Larcker (1981).

En la tabla 2 se muestra que los valores obtenidos cumplen con los indicadores antes mencionados, sin embargo, se presenta la variable de extroversión cuyos valores para alfa y omega no llegan a 0.7 , pero el valor de la media extraída es favorable, debido a esto se decide conservar dicho factor. 
Sánchez Tovar, Yesenia; Macías García, Miguel Ángel; Mendoza Flores, José Esteban Diferencias en los determinantes del éxito en el emprendimiento en México, una perspectiva de género

\begin{tabular}{lllll}
\multicolumn{5}{c}{ Tabla 2} \\
\multicolumn{2}{c}{ Validez convergente de los constructos } \\
\hline Variable & & $\alpha$ & $\Omega$ & AVE \\
\hline Motivación & MO & 0.780 & 0.782 & 0.642 \\
Apertura & AP & 0.855 & 0.868 & 0.689 \\
Extroversión & EV & 0.645 & 0.667 & 0.509 \\
Conciencia & CO & 0.870 & 0.876 & 0.703 \\
Habilidades y conocimiento & HCE & 0.839 & 0.842 & 0.520 \\
Conocimiento del entorno & CE & 0.758 & 0.763 & 0.618 \\
Éxito emprendedor & EX & 0.941 & 0.928 & 0.683 \\
\hline
\end{tabular}

Fuente: Elaboración propia mediante Amos 21.0

Por último, se presentan los resultados de la validez discriminante para finalizar con el análisis del modelo de medida, mediante este análisis se comprueba que las variables del estudio son distintas entre sí, dicho evaluación se realiza con la comparación de las varianzas medias extraídas (AVE) de cada variable con las correlaciones al cuadrado inter-constructo asociadas, se debe de cumplir que dichas correlaciones al cuadrado deberán ser menores a las AVE, tabla 3.

\section{Tabla 3}

Validez discriminante del modelo

\begin{tabular}{cccccccc}
\hline & MO & AP & HCE & CE & EV & CO & EX \\
\hline MO & 0.642 & 0.130 & 0.125 & 0.042 & 0.045 & 0.070 & 0.116 \\
\hline AP & & 0.689 & 0.305 & 0.062 & 0.355 & 0.295 & 0.105 \\
\hline HCE & & 0.520 & 0.086 & 0.217 & 0.214 & 0.033 \\
\hline CE & & & 0.683 & 0.086 & 0.041 & 0.099 \\
\hline EV & & & 0.618 & 0.140 & 0.067 \\
\hline CO & & & & & 0.703 & 0.010 \\
\hline EX & & & & & & & \\
\hline
\end{tabular}

Fuente. Elaboración propia 
En efecto, en los resultados obtenidos se identifica que se cumple lo anteriormente expuesto, por lo que se logra validar la pertenencia discriminante de los indicadores para cada variable, dado los resultados de cada proceso de validación antes expuesto se da por comprobada la fiabilidad de las medidas para las variables de personalidad, habilidades gerenciales, conocimientos del entorno y el éxito emprendedor.

\section{Factores determinantes del éxito emprendedor: Resultados de la investigación}

Después de haber comprobado la fiabilidad de la escala a través del modelo de medida, corresponde evaluar el modelo estructural propuesto, mediante el cual se pretende contrastar las principales hipótesis que componen esta investigación, siendo éstas, las que determinan el éxito emprendedor, como la apertura, la motivación de logro y el conocimiento del entorno, fungiendo como variables exógenas del modelo.

Para analizar el efecto causal de los rasgos personales, la motivación, habilidades gerenciales y el conocimiento del entorno en el emprendimiento exitoso, se procedió a estimar el modelo causal, en primera instancia se evaluó el ajuste del modelo mediante las métricas de ajuste absoluto, incremental y de parsimonia.

La Tabla 4 presenta los principales indicadores de ajuste, en cuanto al ajuste absoluto del modelo, se considera aceptable al lograr un GFI índice de bondad de ajuste) de 0.9 el cual es el rango mínimo marcado por Bentler y Bonett, (1980), del mismo modo se obtuvo un buen ajuste en el error del modelo estructural (RMSEA) con un valor de 0.044, cuando la literatura considera adecuados valores menores a 0.05 (Browne y Cudeck, 1993). Por otra parte, el ajuste incremental (CFI, TLI, IFI) también cumple con valores de adecuado ajuste al ser superiores a 0.9 (Hooper et al, 2008; Hair et al, 2010). Para iniciar con este análisis se realiza el ajuste del modelo mediante los indicadores que evalúan el ajuste incremental, absoluto y de parsimonia. El modelo causal que se presenta analiza los efectos de las relaciones de los rasgos de la personalidad, la motivación, las habilidades gerenciales $y$ el conocimiento de entorno con el emprendimiento exitoso.

Tabla 4

Ajuste del modelo

\begin{tabular}{|c|c|c|c|c|}
\hline \multicolumn{2}{|c|}{ Medidas de ajuste } & \multirow[b]{2}{*}{ Incremental } & & \\
\hline Absoluto & & & \multicolumn{2}{|c|}{ Parsimonia } \\
\hline CMIN & 625.869 & CFI 0.943 & $\mathrm{CMIN} / \mathrm{gl}$ & 1.442 \\
\hline GFI & 0.900 & TLI $\quad 0.934$ & & \\
\hline RMSEA & 0.042 & IFI 0.945 & & \\
\hline
\end{tabular}

Fuente: Elaboración propia 
A continuación, se muestran los resultados obtenidos de las hipótesis planteadas, además se muestra el contraste de las relaciones estudiadas que identifican los determinantes del éxito del emprendimiento, resultando en los hombres que el enfoque gerencial es determinante del éxito de emprendimiento a través del factor de conocimiento de entorno con un coeficiente de correlación de $2.485^{* *}$ y un $\mathrm{P}$ valor significativo de 0.013 .
Por otro lado, para las mujeres resultó el enfoque conductual como favorecedor del éxito del emprendimiento, siendo el factor de rasgos de la personalidad con el éxito como hipótesis no rechazada, estos rasgos son la extroversión, la apertura y la conciencia, dicho hipótesis resultó no rechazada con un coeficiente de correlación de $0.363^{* *}$ y un $\mathrm{P}$ valor singnificativo de 0.024 . (Tabla 5)

\section{Tabla 5}

\section{Evaluación de las hipótesis de la investigación}

\begin{tabular}{|c|c|c|c|c|c|c|c|}
\hline \multirow[t]{2}{*}{ Género } & \multicolumn{3}{|c|}{ Hipótesis } & \multirow{2}{*}{$\begin{array}{l}\begin{array}{c}\text { Solución Estan- } \\
\text { darizada }\end{array} \\
0.201\end{array}$} & C.R & $P$ & \multirow[b]{2}{*}{ Rechazada } \\
\hline & H1 & $\mathrm{RP}$ & EX & & 1.336 & 0.181 & \\
\hline \multirow{3}{*}{ Hombres } & H2 & MO & EX & 0.223 & 1.812 & 0.070 & Rechazada \\
\hline & H3 & HCE & EX & -0.043 & -0.332 & 0.740 & Rechazada \\
\hline & H4 & CE & EX & $0.268^{* *}$ & 2.485 & 0.013 & No se rechaza \\
\hline \multirow{4}{*}{ Mujeres } & H1 & RP & EX & $0.363^{\star *}$ & 2.252 & 0.024 & No se rechaza \\
\hline & H2 & MO & EX & 0.217 & 1.798 & 0.072 & Rechazada \\
\hline & H3 & HCE & EX & -0.262 & -1.657 & 0.098 & Rechazada \\
\hline & H4 & CE & EX & 0.212 & 1.773 & 0.076 & Rechazada \\
\hline
\end{tabular}

${ }^{* *} p$ valor $.01{ }^{*} p$ valor .05

Fuente: Elaboración propia

Por último, las variables de motivación y habilidades gerenciales no resultaron como determinante en ambos grupos de análisis, con niveles de no significativos mayores a 0.05 . En el diagrama 1 y 2 pueden observarse los resultados del modelo estructural para los dos grupos de análisis. 
pp. $880-902$

Revista Venezolana de Gerencia, Año 26 No. 94, 2021

\section{Diagrama 1 \\ Modelo estructural estandarizado Hombres}

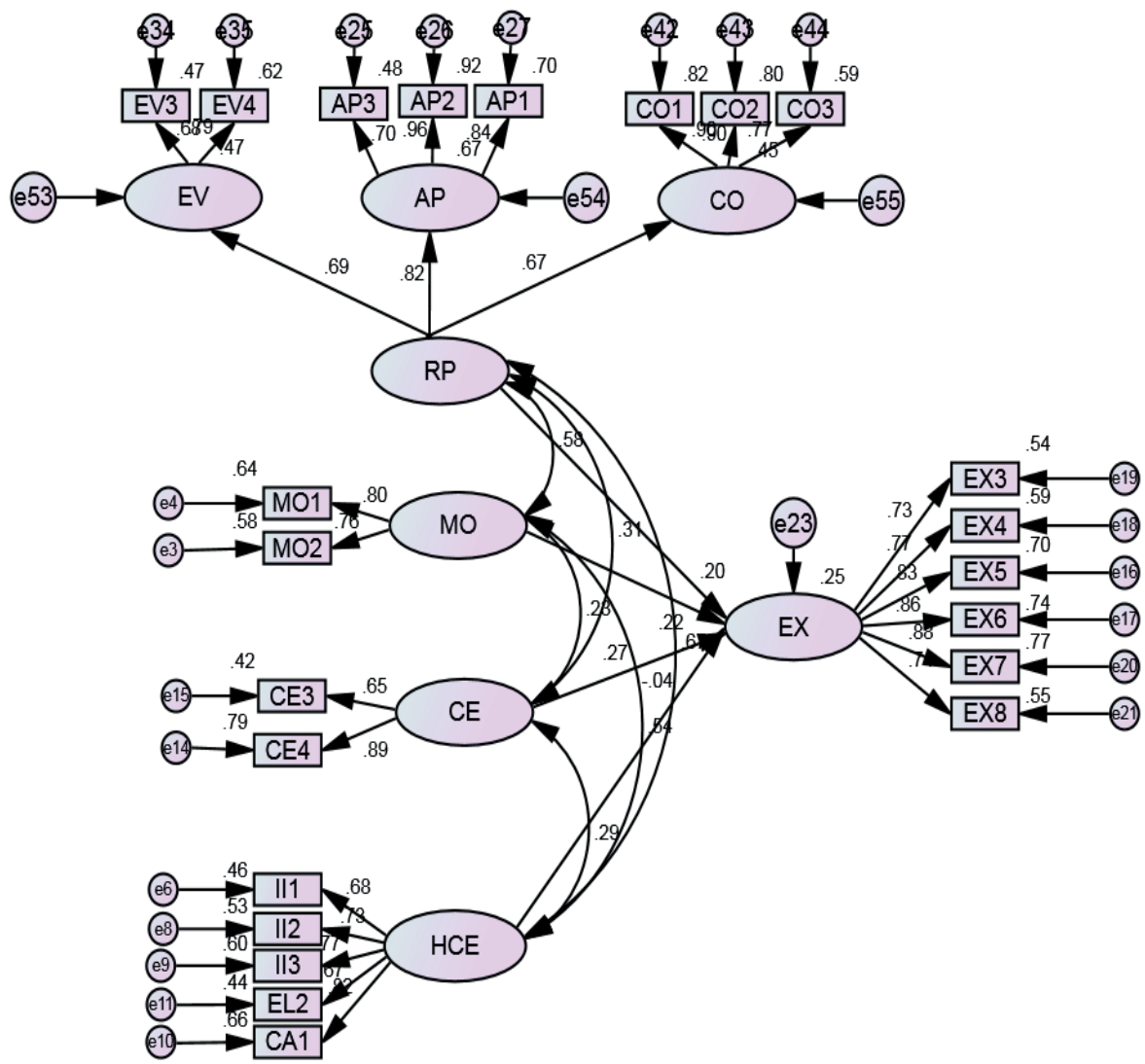

Fuente. Elaboración propia 


\section{Diagrama 2 \\ Modelo estructural estandarizado Mujeres}

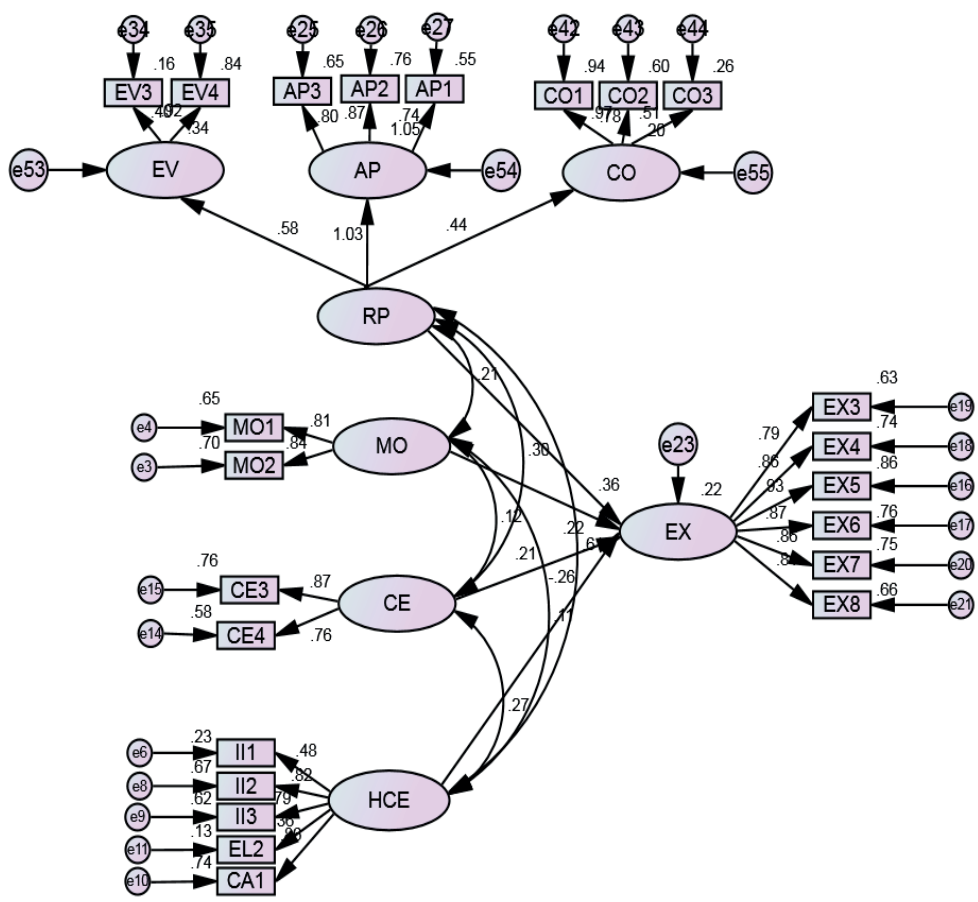

Fuente. Elaboración propia

Los hallazgos de esta investigación muestran que son los elementos psicoconductuales los de mayor peso en la determinación del emprendedor exitoso femenino. En primer lugar, en cuanto a los rasgos personales se pudo concluir que la apertura, la extroversión y la conciencia son los rasgos específicos que favorecen el éxito del emprendedor. Estos resultados son acordes a los encontrados por Rauch y Frese (2007),
Schmitt-Rodermund (2004, 2007), Fine et al, (2012) entre otros.

Aunado a lo anterior se especifica que la extroversión, la apertura y la conciencia fungen como factores que determinan unas buenas relaciones interpersonales y que por rol de género competen más a las mujeres, contrario a los factores de carácter individual como el tomador de riesgos y el locus de control que caracteriza al hombre, como 
indican investigaciones como las de Eagly (2009) y Wieland y Sarin (2012), en este trabajo se encontró que los rasgos de la personalidad mencionados anteriormente favorecen el éxito emprendedor.

También se confirma al revisar la relación del conocimiento del entorno como variable favorecedora del éxito emprendedor de los directivos de género masculino, en estudios como en el de Clarke, et al, (2016) donde se identifica la relevancia de aprovechar el dinamismo del entorno para hacer crecer la empresa, así como el alto nivel de conocimiento de las instituciones permite una ágil apertura de empresas y poder acceder a financiamiento que favorezcan el desarrollo de esta.

Además, se pudo comprobar la brecha de género que argumentan autores como (Santos, Roomi y Liñán, 2016), los factores que resultaron como determinantes del éxito del emprendimiento para las mujeres fueron sus rasgos personales y para los hombres el conocimiento de la estructura institucional, determinados por el conocimiento del entorno empresarial. Sobre todo, las instituciones de financiamiento, en estudios como los de Langowitz y Minniti (2007) y Minniti y Nardone (2007), quienes determinan que las mujeres encuentran más barreras que los hombres para acceder al financiamiento para crear empresas.

Asociado a lo anterior, trabajos como el de Westhead y Solevik (2016) soportan el resultado obtenido sobre el efecto de los conocimientos del entorno emprendedor que en hombres resultó positivo, debido a que la formación emprendedora no se hace llegar de manera adecuada a las mujeres y es más bien sus características personales las que provocan que sus emprendimientos sean exitosos como lo demuestra este trabajo.

\section{Conclusiones}

tuvo por objetivo analizar los factores conductuales y gerenciales determinantes del emprendimiento exitoso desde un enfoque de género. Para el logro de dicho objetivo se realizó una investigación empírica con 255 emprendedores del norte de México.

Esta investigación se centró en los dos grandes enfoques que rodean al individuo al momento de emprender, en el enfoque conductual se enfatiza en la personalidad del sujeto como determinante del desarrollo exitoso de su emprendimiento, en tanto que el enfoque gerencial implica que el individuo debe poseer una serie de habilidades, experiencia y conocimiento de su entorno para lograr el éxito. Los hallazgos encontrados pudieron identificar que el éxito de un emprendimiento no depende de los mismos factores ni de los mismos enfoques en el caso de emprendimientos liderados por hombres que por mujeres.

En primer lugar, se observó que en el caso de los hombres el determinante para lograr el éxito en el emprendimiento se centra en el enfoque gerencial, específicamente en el conocimiento del entorno que adquiere con independencia de la personalidad del individuo. Lo anterior implica que el género masculino suele estudiar los elementos políticos, sociales, culturales y ambientales que rodean su negocio para tomar las decisiones de gerencia, aplicando así una racionalidad mayor a sus decisiones. En el caso de las mujeres, los determinantes del éxito radican en su personalidad, es decir en el enfoque conductual en donde la 
Sánchez Tovar, Yesenia; Macías García, Miguel Ángel; Mendoza Flores, José Esteban Diferencias en los determinantes del éxito en el emprendimiento en México, una perspectiva de género

apertura, la conciencia y la extroversión se conjugan para crear una personalidad perseverante, comunicativa y capaz de adaptarse al entorno.

Estas diferencias traen consigo implicaciones interesantes para el Estado. Se sabe que el emprendimiento es uno de los caminos al desarrollo de un territorio, por lo que el gobierno de dichas regiones ha instado a las autoridades educativas a promover el desarrollo de habilidades emprendedoras que cambien la visión de los jóvenes en cuanto a las oportunidades económicas para no cerrarse a la búsqueda de contrataciones 0 empleos. Por tal motivo las instituciones educativas deben considerar que factores deben impulsarse para desarrollar la capacidad de emprendimiento. No obstante, se debe tener claro que los factores individuales del emprendimiento deben obedecer a una conjunción de una personalidad con rasgos y motivantes apropiados; y una colección de habilidades y conocimientos. No se puede impulsar el éxito del individuo con la ausencia de las características apropiadas.

Lo anterior implica que deben brindarse a las mujeres las oportunidades para preparase académicamente y a los hombres inculcarse la capacidad de conocimiento de la personalidad, eliminando los estereotipos de ciertos rasgos que son asociados solo con las mujeres, para de esta forma lograr un equilibrio de oportunidades que desemboque en un emprendimiento duradero y exitoso.

\section{Referencias Bibliográficas}

Abu-Saifan, S. (2012). Social entrepreneurship: definition and boundaries. Technology innovation management review, 2(2), 22-27. http://doi.org/10.22215/timreview/523

Acs, Z. J., y Audretsch, D. B. (1990). The determinants of small-firm growth in US manufacturing. Applied Economics, 22(2), 143153 https://doi. org/10.1080/00036849000000058

Acs, Z. J., y Mueller, P. (2008). Employment effects of business dynamics: Mice, gazelles and elephants. Small Business Economics, 30(1), 85-100. https:// doi.org/10.1007/s11187-007-9052-3

Amit, R., MacCrimmon, K., Zietsma, C., y Oesch, J. (2000). Does money matter? Wealth attainment as the motive for initiating growth-oriented technology ventures. Journal of Business Venturing, 16(2), 119143. https://doi.org/10.1016/S08839026(99)00044-0

Anderson, G., Keith, M. J., Francisco, J., y Fox, S. (2018). The Effect of Software Team Personality Composition on Learning and Performance: Making the" Dream" Team. In Proceedings of the 51st Hawaii International Conference on System Sciences. https://doi. org/10.24251/HICSS.2018.059

Aubert, P., Caroli, E., y Roger, M. (2006). New technologies, organisation and age: firm-level evidence. The Economic Journal, 116(509), F73-F93. $\quad$ https://doi.org/10.1111/ j.1468-0297.2006.01065.x

Barba, V. (2000) La motivación para crear una empresa y su efecto sobre la satisfacción del empresario (Tesis Doctoral), Universidad de Castilla La Mancha, España. https://dialnet.unirioja.es/servlet/ tesis? codigo $=24136$

Barrick, M. R., Mount, M. K., \& Judge, T. A. (2001). Personality and performance at the beginning of 
the new millennium: What do we know and where do we go next? International Journal of Selection and assessment, 9(1-2), 9-30. https://doi. org/10.1111/1468-2389.00160

Bentler, P. M. y Bonett, D. G. (1980) Significance tests and goodnessof-fit in the analysis of covariance structures. Psychological Bulletin, 88(1) 588-600. https://doi. org/10.1037/0033-2909.88.3.588

Blonigen, B., Harbaugh, W., Singell, L., Horner, R., Irvin, L., y Smolkowski, L. (2008). Application of Economic Analysis to School-Wide Positive Behavior Support (SWPBS) Programs. Journal ofPositiveBehavior Interventions, 10(1), 5-19. http://doi. org/10.1177/1098300707311366

Brandstätter, H. (2011). Personality aspects of entrepreneurship: A look at five meta-analyses. Personality and individual differences, 51(3), 222-230. https://doi.org/10.1016/j. paid.2010.07.007

Browne, M. W., y Cudeck, R. (1993). Alternative ways of assessing model fit. Sociological Methods \& Research, 154(1), 136-136. https://doi.org/10.11 77/0049124192021002005

Bulut, C., y Culha, O. (2010). The effects of organizational training on organizational commitment. International Journal of Training and Development, 14(4), 309322. https://doi.org/10.1111/j.14682419.2010.00360.x

Busato, V. V., Prins, F. J., Elshout, J. J., y Hamaker, C. (2000). Intellectual ability, learning style, personality, achievement motivation and academic success of psychology students in higher education. Personality and Individual differences, 29(6), 1057-1068.

Bygrave, W. D., y Hofer, C. W. (1992).
Theorizing about entrepreneurship. Entrepreneurship theory and Practice, 16(2), 13-22. https://doi.org /10.1177/104225879201600203

Carlisle, E., y Flynn, D. (2005). Small business survival in China: Guanxi, legitimacy, and social capital. Journal of Developmental Entrepreneurship, 10(1), 79-96. https://doi.org/10.1142/ $\underline{\mathrm{S} 1084946705000070}$

Chell, E. (2008). The nascent entrepreneur, business development and the role of human resources. International handbook of entrepreneurship and HRM. Elgar, Inglaterra. https://doi.org/10.4337/97 $\underline{81848441224.00008}$

Ciavarella, M. A., Buchholtz, A. K., Riordan, C. M., Gatewood, R. D., y Stokes, G. S. (2004). The Big Five and venture survival: Is there a linkage? Journal of Business Venturing, 19(4), 465-483. https://doi. org/10.1016/j.jbusvent.2003.03.001

Clarke, L., McFarland, J., Octaviano, C., van Ruijven, B., Beach, R., Daenzer, K.,.. y Rodriguez, A. M. L. (2016). Long-term abatement potential and current policy trajectories in Latin American countries. Energy Economics, 56(1), 513-525. https:// doi.org/10.1016/j.eneco.2016.01.011

Codina, A. (2005). ¿Qué hacen los directivos y qué habilidades necesitan para un trabajo efectivo. Resumen y análisis de Investigación. México. https://bit.ly/3xbBSzn

Covin, J. G., Green, K. M., y Slevin, D. P. (2006). Strategic process effects on the entrepreneurial orientationsales growth rate relationship. Entrepreneurship theory and practice, 30(1), 57-81. https://doi.org/10.1111/ j.1540-6520.2006.00110.x

Cuervo A., Ribeiro, D., y Roig, S. (2007). Entrepreneurship: Concepts, Theory 
Sánchez Tovar, Yesenia; Macías García, Miguel Ángel; Mendoza Flores, José Esteban Diferencias en los determinantes del éxito en el emprendimiento en México, una perspectiva de género

and perspective. Springer, New York. https://doi.org/10.1007/978-3-54048543-8 1

De Miguel Pascual, R., ParrattFernández, S. y Berganza, R. (2019). Las percepciones de las mujeres periodistas sobre su trabajo. La variable género en la cultura profesional. Revista Latina de Comunicación Social, 1818-1833. https://doi.org/10.4185/RLCS-20191412-95

D’Enbeau, S., Villamil, A., y Helens-Hart, R. (2015). Transcending work-life tensions: A transnational feminist analysis of work and gender in the Middle East, North Africa, and India. Women's Studies in Communication, 38(3), 273-294. https://doi.org/10.10 80/07491409.2015.1062838

Dinur, A.R. (2011). Common and uncommon sense in managerial decision making under task uncertainty. Management Decision, 49(5), 694-709. https://doi. org/10.1108/00251741111130797

Eagly, A. H. (2009). The his and hers of prosocial behavior: An examination of the social psychology of gender. American Psychologist, 64(8), 644. https://doi.org/10.1037/0003066X.64.8.644

Ensley, M. D., Hmieleski, K. M., y Pearce, C. L. (2006). The importance of vertical and shared leadership within new venture top management teams: Implications for the performance of startups. The leadership quarterly, 17(3), 217-231. https://doi. org/10.1016/j.leaqua.2006.02.002

European Certificate in Entrepreneurship. (2006). Are you an entrepreneur? User Guide. Consultado en http:// www.ecent.org

Fayolle, A., Linán, F. y Moriano, J. A. (2014). Beyond entrepreneurial intentions: values and motivations in Entrepreneurship. International Entrepreneurship Management Journal, 10, 679-689. https://doi. org/10.1007/s11365-014-0306-7

Fine, et al, (2012). Psychological Predictors of Successful Entrepreneurship in China: An Empirical Study. International Journal of Management, 9(1), 279-292. https://bit.ly/3nc234g

Formichella, M. M. (2004). El concepto de emprendimiento y su relación con el empleo, la educación y el desarrollo local. Conference: VII Congreso Nacional e internacional de Administración, y XI CONAMerco (Congreso de Administración del MERCOSUR). https://bit.ly/3sCH6R6

Fornell, C., y Larcker, D. F. (1981). Structural equation models with unobservable variables and measurement error: Algebra and statistics. Journal of marketing research, https://doi.org/382$388.10 .2307 / 3150980$

Goldberg, L. R. (1992). The development of markers for the Big-Five factor structure. Psychological Assessment, 4, 26-42

González, M. (2001). Actitudes y motivaciones hacia el trabajo y la creación de empresas según género. Boletín Económico ICE, 2709, 21-29.

Hair, J. F., Black, W. C., Babin, B. J., y Anderson, R. E. (2010). Multivariate Data Analysis. Pearson. Estados Unidos de América.

Hajek, O., Nekolova, J., y Novosak, J. (2015). Determinants of New Business Formation - Some Lessons from the Czech Republic. Economics and Sociology, 8(1), 147-156.

Hájek, O., Nekolová, J., y Novosák, J. (2015). Determinants of new 
business formation-some lessons from the Czech Republic. Economics and Sociology, 8(1). 147-156. https://doi.org/10.14254/2071789X.2015/8-1/11

Heise, D. R., y Bohrnstedt, G. W. (1970). Validity, invalidity, and reliability. Sociological methodology, 2(1), $\quad$ https://doi.org/104$\underline{129,10.2307 / 270785}$

Herron, L., y Robinson Jr, R. B. (1993). A structural model of the effects of entrepreneurial characteristics on venture performance. Journal of business venturing, 8(3), 281294 https://doi.org/10.1016/08839026(93)90032-Z

Hill, C. y Jones, G. (2011). Administración Estratégica. Un enfoque integral. (9a ed.). Mexico, Mexico: Cengage Learning.

Hooper, D., Coughlan, J., y Mullen, M. (2008). Structural equation modelling: Guidelines for determining model fit. Articles, 2(1), 53-60, https:// doi.org/10.21427/D7CF7R.

Ihrig, M., Zu Knyphausen-Aufseß, D., y O'Gorman, C. (2006). The knowledgebased approach to entrepreneurship: linking the entrepreneurial process to the dynamic evolution of knowledge. International Journal of Knowledge Management Studies, 1(1-2), 38-58, https://doi.org/10.1504/ IJKMS.2006.008844.

Instituto Nacional de Estadística y Geografía (INEGI, 2016). https:// www.inegi.org. $\mathrm{mxl}$

Jackson, T., Amaeshi, K., y Yavuz, S. (2008). Untangling African indigenous management: Multiple influences on the success of SMEs in Kenya. Journal of World Business, 43(4), 400-416.

John, O. P., Naumann, L. P., y Soto,
C. J. (2008). Paradigm shift to the integrative big five trait taxonomy. Handbook of personality: Theory and research, 3(2), 114-158. https://doi. org/10.1016/j.jwb.2008.03.002.

Kolb, D. A., Rubin, I. M., y Osland, J. S. (2007). Organizational behavior: An experiential approach. Englewood Cliffs, NJ: Prentice Hall.

Laforet, S. (2013). Organizational innovation outcomes in SMEs: Effects of age, size, and sector. Journal of World business, 48(4), 490-502, https://doi.org/10.1016/j. jwb.2012.09.005

Langowitz, N. y Minniti, M. (2007). The entrepreneurial propensity of women. Entrepreneurship theory and practice, 31(3), 341364 https://doi.org/10.1111/i.15406520.2007.00177.x.

Leutner, F., Ahmetoglu, G., Akhtar, R., y Chamorro-Premuzic, T. (2014). The relationship between the entrepreneurial personality and the Big Five personality traits. Personality and individual differences, 63(1), 58-63. https://doi.org/10.1016/j. paid.2014.01.042

Lucas, R. E., \& Donnellan, M. B. (2011). Personality development across the life span: Longitudinal analyses with a national sample from Germany. Journal of Personality and Social Psychology, 101(4), 847-861. https://doi.org/10.1037/ $\underline{\mathrm{a} 0024298}$

Marlow, S. (1997). Self-employed women-new opportunities, old challenges? Entrepreneurship \& Regional Development, 9(3), 199210.

McClelland, D. (1961). The Achieving Society. New York: The Free Press.

Migliore, L.A. (2011). Relation between big 
Sánchez Tovar, Yesenia; Macias García, Miguel Ángel; Mendoza Flores, José Esteban Diferencias en los determinantes del éxito en el emprendimiento en México, una perspectiva de género

five personality traits and Hofstede's cultural dimensions: Samples from the USA and India. Cross Cultural Management: An International Journal, 18(1), 38-54, https://doi. org/10.1108/13527601111104287

Minniti, M. (2009). Gender issues in entrepreneurship. Foundations and Trends in Entrepreneurship, 5(7-8), 497-620, https://ssrn.com/ abstract $=1543464$.

Minniti, M. y Bygrave, W. (2001). A dynamic model of entrepreneurial learning. Entrepreneurship Theory and Practice, 25(3), 5-16, https://doi. org/10.1177/104225870102500301

Minniti, M., y Nardone, C. (2007). Being in someone else's shoes: the role of gender in nascent entrepreneurship. Small business economics, 28(23), 223-238 https://doi.org/10.1007/ s11187-006-9017-y

Montes de Oca Rojas, Y. (2020). Perspectivas del emprendimiento social y redes socioproductivas de pequeños productores en Venezuela. Revista De Ciencias Sociales, 26(1), 300-312. https://doi. org/10.31876/rcs.v26i1.31326

Munkejord, M. C. (2017). Becoming Spatially Embedded: Findings from a study on rural immigrant entrepreneurship in Norway. Entrepreneurial Business and Economics Review, 5(1), 111130, $\quad$ https://doi.org/10.15678/ EBER.2017.050107.

Murugesan, R., y Jayavelu, R. (2017). The influence of big five personality traits and self-efficacy on entrepreneurial intention: The role of gender. Journal of entrepreneurship and innovation in emerging economies, 3(1), 41-61.

Naktiyok, A., Karabey, C. N., y Gulluce, A. C. (2010). Entrepreneurial selfefficacy and entrepreneurial intention: the Turkish case. International Entrepreneurship and Management Journal, 6(4), 419-435, https://doi. org/10.1007/s11365-009-0123-6.

Perren, L. (1999). Factors in the growth of micro-enterprises (Part 1): Developing a framework. Journal of small business and enterprise development, 6(4), 366-385 https://doi.org/10.1108/ EUM0000000006691.

Pires, C., Sarkar, S. Y Carvalho, L. (2008). Innovation in services how different from manufacturing? The Service Industries Journal, 28(10), 1339-1356, https://doi. org/10.1080/02642060802317812

Rauch, A., y Frese, M. (2007). Let's put the person back into entrepreneurship research: A metaanalysis on the relationship between business owners' personality traits, business creation, and business success. European Journal of Work and Organizational Psychology, 16(4), 353-385, https://doi. org/10.1080/13594320701595438

Reisinger, S., y Lehner, J. M. (2015). Navigating a family business through a changing environment: findings from a longitudinal study. Review of Managerial Science, 9(2), 411-429, https://doi.org/10.1007/s11846-0140163-3.

Roberts, B., y DelVecchio, W. (2000). The Rank-Order Consistency of Personality Traits from Childhood to Old Age: A Quantitative Review of Longitudinal Studies. Psychological Bulletin, 126(1), 3-25 https://bit. Iy/3sNc4WB

Runyan, R. C., Huddleston, P. y Swinney, J. (2006). Entrepreneurial orientation and social capital as small firm strategies: A study of gender differences from a resourcebased view. The International 
Entrepreneurship and Management Journal, 2(4), 455 https://doi. org/10.1007/s 11365-006-0010-3 .

Saavedra-García, M. L., Tapia-Sánchez, B., y de los Ángeles Aguilar-Anaya, M. (2016). La gestión financiera en las pymes del Distrito Federal, México. Revista Perspectiva Empresarial, 3(2), 55-69, https://doi.org/10.16967/ rpe.v3n2a5.

Şahin, F., Karadağ, H., y Tuncer, B. (2019). Big five personality traits, entrepreneurial selfefficacy and entrepreneurial intention: A configurational approach. International Journal of Entrepreneurial Behavior \& Research.

Santos, F. J., Roomi, M. A., y Liñán, F. (2016). About gender differences and the social environment in the development of entrepreneurial intentions. Journal of Small Business Management, 54(1), 4966, https://www.tandfonline.com/doi/ abs/10.1111/jsbm.12129

Schefczyk, M. (2001). Determinants of success of German venture capital investments. Interfaces, 31(5), 43-61, https://doi.org/10.1287/ inte.31.5.43.9656.

Schmitt-Rodermund, E. (2004). Pathways to successful entrepreneurship: Parenting, personality, early entrepreneurial competence, and interests. Journal of vocational behavior, 65(3), 498-518, https://doi. org/10.1016/j.jvb.2003.10.007.

Schmitt-Rodermund, E. (2007). The long way to entrepreneurship: Personality, parenting, early interests, and competencies as precursors for entrepreneurial activity among the "Termites." In R. K. Silbereisen y R. M. Lerner (Eds.), Approaches to positive youth development. London, England: Sage.
Sexton, D. L., \& Bowman-Upton, N. (1990). Female and male entrepreneurs: Psychological characteristics and their role in gender-related discrimination. Journal of business venturing, 5(1), 29-36.

Shapero, A. (1984). The entrepreneurial event. In C. A. Kent (Ed.), The environment for entrepreneurship. Lexington, Mass.

Shepherd, D. A., Patzelt, H. y Haynie, J. M. (2010). Entrepreneurial spirals: Deviation-amplifying loops of an entrepreneurial mindset and organizational culture. Entrepreneurship theory and practice, 34(1), 59-82.

Sung, S. Y., y Choi, J. N. (2009). Do big five personality factors affect individual creativity? The moderating role of extrinsic motivation. Social Behavior and Personality: an international journal, 37(7), 941-956.

Sunindijo, R. Y., Hadikusumo, B. H. y Ogunlana, S. (2007). Emotional intelligence and leadership styles in construction project management. Journal of management in engineering, 23(4), 166-170, https:// bit.ly/2Pcay $2 \mathrm{C}$

Van Riel, A.C.R., Lemmink, J. y Ouwersloot, H. (2004). Hightechnology service innovation success: A decision-making perspective. Product Innovation Management, 21, 348-359, https://doi.org/10.1111/j.07376782.2004.00087.x.

Veciana, J.M. (1999). Creación de Empresas como programa de investigación Científica. Revista Europea de Dirección y Economía de la Empresa, 8(3) 11-36. https://dialnet.unirioja.es/servlet/ articulo? codigo $=497564$. 
Sánchez Tovar, Yesenia; Macías García, Miguel Ángel; Mendoza Flores, José Esteban Diferencias en los determinantes del éxito en el emprendimiento en México, una perspectiva de género

Veciana, J.M. (2005). La Creación de Empresas: un enfoque gerencial. Colección de estudios económicos, La Caixa, Barcelona.

Wang, C. (2008). Entrepreneurial orientation, learning orientation, and firm performance. Entrepreneurship Theory and Practice, 32(4), 635657, https://doi.org/10.1111/j.1540$6520.2008 .00246 . x$

Watson, D. y Clark, L. A. (1997). Extraversion and its positive emotional core. In Handbook of personality psychology. Academic Press.

Westhead, P. y Solesvik, M. Z. (2016). Entrepreneurship education and entrepreneurial intention: do female students benefit? International Small Business Journal, 34(8), 979-1003, https://doi. org/10.1177/0266242615612534

Wieland, A., y Sarin, R. (2012). Domain specificity of sex differences in competition. Journal of Economic Behavior y Organization, 83(1), 151-157., $\quad$ https://doi.org/10.1016/j. jebo.2011.06.019

Wiklund, J. y Shepherd, D. (2005). Entrepreneurial orientation and small business performance: a configurational approach. Journal of business venturing, 20(1), 71-91, https://doi.org/10.1016/i. jbusvent.2004.01.001 